\title{
Resiliência Comunitária: Um estudo de Revisão Integrativa da Literatura
}

\author{
Ana Tercila Campos Oliveira*, 1 \\ Orcid.org/0000-0001-7254-3969 \\ Normanda Araujo de Morais ${ }^{1}$ \\ Orcid.org/0000-0003-3156-4688
}

${ }^{1}$ Universidade de Fortaleza, Fortaleza, CE, Brasil

\begin{abstract}
Resumo
Realizou-se uma revisão integrativa da literatura (nacional e internacional) sobre o tema da resiliência comunitária (RC), no período de 2009 a 2015. Especificamente, buscou-se descrever os conceitos de $\mathrm{RC}$ trabalhados nos artigos, as principais adversidades citadas e os fatores relacionados à RC. A busca foi feita no SciELO e no Portal de Periódicos Capes e, a partir dos critérios de inclusão e exclusão, identificaram-se 34 artigos. Predominaram os artigos na língua inglesa, sobretudo norte-americanos, publicados em 2013, de método qualitativo e baseado em entrevistas. Sobre o conceito de RC, verificouse que esta pode ser vista como um atributo (capacidade, habilidade) e enquanto processo. As principais adversidades estudadas são os desastres (furacões, terremotos, enchentes, ataques terroristas e conflitos armados). E três fatores estão relacionados à RC: capital social, laços culturais e a infraestrutura que apoia o desenvolvimento dos membros da comunidade. Por fim, sublinha-se o grande potencial de contribuição teórica e prática desse conceito para a superação de adversidades comuns ao contexto comunitário brasileiro.
\end{abstract}

Palavras-chave: Resiliência comunitária, comunidade, capital social.

\section{Community Resilience: An Integrative Literature Review study}

\begin{abstract}
A national and international integrative literature review on the topic of community resilience (CR) was performed, focusing on the period from 2009 to 2015. Specifically, we sought to describe the concepts of CR developed in articles, the main adversities cited and the factors related to CR. The search was conducted using the SciELO database and Portal de Periódicos Capes, and from the inclusion and exclusion criteria, 34 articles were identified. Most articles were found in the English language, especially from North America, published in 2013, and used a qualitative method, based on interviews. With regard to the concept of CR, it was found that this can be seen as an attribute (ability and skill)

* Endereço para correspondência: Universidade de Fortaleza, Programa de Pós-Graduação em Psicologia, Avenida Washington Soares, 1321, Edson Queiroz, Fortaleza, CE, Brasil 60.811-905. E-mail: tercilacampos@ hotmail.com

Agradecimento: As autoras agradecem à Fundação Cearense de Apoio ao Desenvolvimento Científico e Tecnológico (FUNCAP) e ao Conselho Nacional de Desenvolvimento Científico e Tecnológico (CNPQ), respectivamente, pelas Bolsas de Mestrado e de Produtividade II em Pesquisa.
\end{abstract}


and a process. The main adversities that were studied were disasters (hurricanes, earthquakes, floods, terrorist attacks and armed conflicts). There are three factors related to CR: social capital, cultural ties and the infrastructure that supports the development of community members. Finally, the great potential of theoretical and practical contribution of this concept to the overcoming of adversities common to the Brazilian community context is underlined.

Keywords: Community resilience, community, social capital.

\section{Resiliencia Comunitaria: Un estudio de Revisión Integradora de la Literatura}

\section{Resumen}

Se llevó a cabo una revisión integradora de la literatura (nacional y internacional) sobre el tema de la resiliencia comunitaria (RC), en el periodo desde 2009 hasta 2015. Específicamente, buscamos describir los conceptos de RC utilizados en los artículos, las principales adversidades y factores relacionados con la RC. La busqueda se realizó en el SciELO y en el Portal de Periódicos Capes y, a partir de los criterios de inclusión y exclusión, se identificaron 34 artículos. Predominaron los artículos en inglés, especialmente los estadounidenses, publicados en 2013, y el método cualitativo basado en entrevistas. Sobre el concepto de RC, se concluyó que se puede verlo como un atributo (capacidad, habilidad) y también como un proceso. Las principales dificultades estudiadas fueron los desastres (huracanes, terremotos, inundaciones, ataques terroristas y conflictos armados). Y tres factores fueran relacionados con la RC: el capital social, los lazos culturales y la infraestructura que apoya el desarrollo de los miembros de la comunidad. Por último, se pone en evidencia el gran potencial y contribución teórico y práctico de este concepto para la superación de las adversidades comunes al contexto comunitario brasileño.

Palabras claves: Resiliencia comunitaria, comunidad, capital social.

Desde o início da história da humanidade, comunidades, sociedades e civilizações precisam lançar mão de capacidades adaptativas para sobreviver às grandes mudanças sociais e ambientais. Na contemporaneidade, os desafios têm se multiplicado. A globalização, mudanças climáticas e o crescimento econômico desenfreado favorecem a ocorrência de situações estressoras para indivíduos e comunidades, tais como desastres de causa natural, tecnológica ou humana, guerras, epidemias e desigualdade social. $\mathrm{O}$ termo resiliência tem sido utilizado por muitos especialistas para explicar respostas adaptativas e saudáveis que indivíduos e grupos apresentam a esses desafios, gerando um corpo crescente de pesquisa sobre o assunto (Smith, Moore, Anderson, \& Siderelis, 2012; Wilson, 2012).

A resiliência psicológica foi investigada, inicialmente, a nível individual, na tentativa de compreender como algumas crianças conseguiam desenvolver-se de forma saudável, ape- sar da exposição a situações adversas graves, como transtorno mental dos pais e pobreza extrema. A princípio, a resiliência foi compreendida como atributo inato ou adquirido e as crianças que apresentavam tal fenômeno foram consideradas invulneráveis, ou seja, capazes de suportar adversidades sem sofrer qualquer impacto (Rutter, 1993). Pesquisas mais recentes revelam, no entanto, que a resiliência não pode ser vista de forma estática, mas sim como um processo dinâmico e subjetivo, no qual características individuais se relacionam com os fatores de risco e proteção ${ }^{1}$

\footnotetext{
Os fatores de risco são entendidos como eventos estressores que aumentam a probabilidade do indivíduo apresentar problemas psicológicos, sociais e físicos, enquanto os fatores de proteção seriam as influências que possibilitam respostas mais adaptativas aos riscos (Morais \& Koller, 2011).
} 
encontrados nos contextos em que o sujeito está inserido. Dessa forma, mais contemporaneamente, a resiliência tem sido descrita por muitos pesquisadores como o conjunto de processos sociais e psíquicos que facilitam o enfrentamento de situações adversas, resultando em adaptação e transformação positiva (Morais \& Koller, 2011; Poletto \& Koller, 2008).

Paralelo aos estudos da Psicologia e Psiquiatria sobre resiliência, o conceito ganhou visibilidade em outros campos do conhecimento, principalmente no da Ecologia. Em 1973, Holling publicou a tese "Resilience and Stability of Ecological Systems" sobre resiliência ecológica, na qual o termo foi usado para descrever a habilidade de um ecossistema para absorver a mudança e adaptar-se a ela, mantendo seu estado existente de funcionamento (Boon, Cottrell, King, Stevenson, \& Millar, 2012). A visão ecossistêmica de resiliência percebe o indivíduo incorporado a uma teia de complexos relacionamentos, de modo que o individual, o familiar e o meio ambiente maior estão interconectados e fatores de cada esfera contribuem para o processo de enfrentamento às adversidades (Kirmayer, Sehdev, Whitley, Dandeneau, \& Isaac, 2009). A partir do surgimento dessa visão, o conceito de resiliência tem sido aplicado não apenas para entender capacidades adaptativas apresentadas por indivíduos, mas também por comunidades humanas e sociedades maiores (Norris, Stevens, Pfefferbaum, Wyche, \& Pfefferbaum, 2008).

Apesar de resiliência comunitária não ser um construto proveniente da Psicologia Comunitária, é possível perceber semelhanças entre suas propostas e preocupações. A Psicologia Comunitária é área da Psicologia que busca compreender a vida cotidiana e concreta das pessoas em uma dada realidade sociohistórica e como esse modo de vida influencia e é influenciado por seus moradores. Um dos principais objetivos da Psicologia Comunitária é contribuir para o desenvolvimento comunitário, que ocorre por meio da conscientização e do fortalecimento de seus moradores, possibilitando o desenvolvimento de seus próprios recursos e potencialidades. Esse processo facilita a organização e ação participativa e compartilhada dos moradores em direção ao enfrentamento e resolução de seus problemas, a autonomia e superação das desigualdades sociais (Góis, 2008; Montero, 1984; Quintal, 2016). Uma vez que a resiliência comunitária consiste nos processos grupais de enfrentamento e adaptação aos desafios coletivos, seu estudo tem o potencial de trazer contribuições para a Psicologia Comunitária, sobretudo se aplicado às intervenções em situações de adversidades crônicas, como a seca, a desigualdade social, violência urbana, entre tantas outras encontradas no contexto brasileiro e latino-americano.

A investigação do conceito de resiliência comunitária na literatura evidencia a existência de duas tradições teóricas, uma latino-americana e uma norte-americana. A latino-americana é representada pelo pesquisador argentino Élbio Néstor Suárez Ojeda. Sua concepção de resiliência comunitária tornou-se mais acessível para pesquisadores brasileiros, pois alguns livros contendo capítulos escritos por ele possuem versão publicada em português (e.g. Ojeda, 2005; Ojeda, La Jara, \& Márquez, 2007). Os artigos da tradição norte-americana, por sua vez, são comparativamente menos citados na literatura nacional, fato que também pode estar relacionado à menor acessibilidade aos textos de língua inglesa, bem como ao fato de se tratar de um tema de interesse bastante recente no contexto brasileiro. Além disso, merece destaque o fato de que, embora seja possível perceber semelhanças entre o que é proposto pelas correntes teóricas da resiliência comunitária (latino-americana e norte-americana), os estudos de uma não se referem a outra.

Os estudos e projetos de promoção de resiliência realizados na América Latina tendem a focar populações marcadas pela pobreza e discriminação, com intervenções orientadas para um sentido social, uma vez que o continente é propenso a diversos desastres tanto por suas condições geográficas quanto por suas condições sociais (Ojeda, 2005; Ojeda et al., 2007). Nesse sentido, Ojeda et al. (2007) afirma que a contribuição latino-americana para o conceito de resiliência é maior no tocante ao enfoque coletivo por estar mais enraizada na epidemiologia social 
do que nos processos de saúde-doença estudados classicamente.

Ojeda (2005) reconhece o dano sofrido pela comunidade causado por um desastre ou catástrofe, mas enfatiza que essa situação dolorosa pode também significar a mobilização de recursos e capacidades solidárias da população para promover transformações físicas e sociais na comunidade. Como se as comunidades contassem com um "escudo protetor", que emerge de suas próprias condições de vida e valores e permite uma metabolização do evento negativo e a possibilidade de construir a partir dele. Através da avaliação da resiliência em diversas comunidades que sofreram desastres, o autor observou as condições que aparecem com maior frequência naquelas que se sobrepõem com mais rapidez e sucesso ao risco e identificou os pilares da resiliência comunitária, a saber: solidariedade, honestidade estatal, identidade cultural, autoestima coletiva e humor social (Ojeda et al., 2007).

A solidariedade é percebida por meio da adesão à causa grupal, com a finalidade de superar as adversidades e conquistar benefícios comuns a todos. Honestidade estatal é vista através da confiança dos membros da comunidade que seus governantes administram a 'coisa pública' de forma honesta e transparente. Identidade cultural diz respeito ao reconhecimento e valorização do que é próprio de uma determinada cultura, incluindo costumes, valores, danças, canções e expressões idiomáticas, o que proporciona aos membros um sentindo de pertença. $\mathrm{O}$ humor social é compreendido como a capacidade de alguns povos de encontrar a comédia na própria tragédia, estratégia que contribui para a aceitação da desgraça comum, favorecendo um distanciamento do problema e possibilitando sua resolução. Autoestima coletiva refere-se à atitude e sentimento de orgulho que o indivíduo tem pelo lugar onde mora, incluindo o amor por sua terra, a consciência de suas belezas naturais e a identificação com determinados costumes e produções humanas significativas.

As produções científicas acerca da resiliência comunitária fora do contexto latino-americano tendem a seguir uma concepção norte-americana, a qual tem como marco conceitual a publicação de um artigo produzido por Fran Norris e colaboradores em 2008. O artigo apresenta uma teoria de resiliência que engloba o entendimento contemporâneo de estresse, adaptação, bem-estar e recursos dinâmicos. De acordo com Norris et al. (2008), resiliência comunitária é o processo que vincula uma rede de capacidades adaptativas (recursos com atributos dinâmicos) da comunidade a uma trajetória positiva de funcionalidade e adaptação após uma perturbação ou adversidade. $\mathrm{O}$ resultado do processo de resiliência é a adaptação comunitária, manifestada pelo bem-estar da população, definido como alto e não díspares níveis de saúde mental e comportamental, funcionamento adequado dos diversos papéis sociais e qualidade de vida (como as pessoas em geral se sentem a respeito de suas vidas como um todo) satifatórios. Essa definição vincula o conceito de resiliência às preocupações tradicionais do campo da saúde pública e saúde mental.

Os atributos dinâmicos dos recursos encontrados em uma comunidade são: robustez, quando o recurso é forte e apresenta baixa probabilidade de deterioração; redundância, variedade e abundância de recursos, a qual permite que os elementos sejam substituídos, caso haja degradação de algum deles; e rapidez, quão rápido um recurso pode ser acessado e utilizado. Segundo o modelo de resistência e resiliência proposto por Norris et al. (2008), a comunidade pode seguir três caminhos diante da exposição a um evento estressor: resistência, disfunção transitória situacional seguida por um processo de resiliência ou disfunção permanente. O resultado mais provável que uma comunidade apresenta diante de eventos estressores é a disfunção transitória situacional, caracterizada por um período de angústia e sofrimento temporário, que pode ser seguido por processos de resiliência comunitária ou vulnerabilidade. Resiliência ocorre quando os recursos dinâmicos da comunidade são capazes de neutralizar os efeitos dos estressores, permitindo adaptação ao ambiente alterado e retorno ao funcionamento após o período de disfunção transitória. Sendo assim, resiliência comunitária emerge de conjuntos de capacidades adaptativas interligadas, que capturam a combinação dos 
recursos em si com seus atributos dinâmicos robustez, redundância e rapidez. As capacidades adaptativas primárias são: Desenvolvimento Econômico, Capital Social, Informação e Comunicação, e Competência Comunitária (Norris et al., 2008).

O desenvolvimento econômico de uma comunidade fomenta sua resiliência na medida em que oferece volume, diversidade em recursos econômicos e igualdade na distribuição dos mesmos. Comunidades pobres estão mais propensas a apresentar reações psicológicas, sociais e físicas disfuncionais devido à situação de vulnerabilidade que se encontravam antes do desastre. O capital social diz respeito às relações do indivíduo com as redes sociais em que ele está incorporado, que proporcionam suporte social e conexão com a comunidade. Informação e Comunicação acerca dos riscos que as pessoas correm, das direções a serem tomadas, da coordenação de voluntários e dos serviços disponíveis ajudam a elevar os níveis de sobrevivência em caso de emergência.

Competência comunitária refere-se às capacidades de tomada de decisão, ação coletiva significativa e intencional, empoderamento, habilidade de engajamento construtivo em processos de grupo, confiança mútua e disposição para trabalhar pelo bem comum da comunidade (Norris et al., 2008).

No Brasil, a produção científica acerca do tema resiliência comunitária ainda é considerada incipiente. As adversidades vivenciadas pela população brasileira e suas possibilidades de enfrentamento têm sido amplamente discutida pela Psicologia Comunitária, mas somente recentemente a lente da resiliência vem sendo utilizada para analisar tais fenômenos. Alguns estudos que contribuem para o desenvolvimento do estado da arte sobre a resiliência comunitária no contexto brasileiro foram publicados em um livro lançado em 2015 sobre resiliência (Coimbra \& Morais, 2015). No primeiro capítulo que aborda o tema, encontra-se o relato do incêndio da boate Kiss em Santa Maria/RS, a intervenção pós-desastre que foi realizada e os processos de resiliência comunitária observados (Zappe, Yunes, \& Dell'aglio, 2015). O segundo capítulo, por sua vez, trata dos processos de resiliência vivenciados por moradores do sertão cearense diante das dificuldades ocasionadas pelo fenômeno natural da seca (Morais, Figueiredo, Rodrigues, \& Santos, 2015).

O estudo da resiliência comunitária possibilita a identificação de fatores que contribuem para processos coletivos de enfrentamento às adversidades. Trata-se de um fenômeno que pode ser promovido, considerando a responsabilidade tanto da comunidade em si, vista em toda sua complexidade e potencialidade para transformação, quanto dos agentes externos (profissionais, governo e terceiro setor), responsáveis por oferecer recursos físicos, econômicos e sociais que tornem as comunidades mais capacitadas para enfrentar as adversidades e menos vulneráveis à elas (White, Edwards, Farrar, \& Plodinec, 2014). Assim, torna-se evidente a necessidade de ampliar o conhecimento acerca dos fatores que estão sendo considerados importantes para a promoção de resiliência comunitária no Brasil e no mundo para que se possa pensar em estratégias de intervenção e promoção de resiliência eficazes para as comunidades que vivenciam algum tipo de adversidade.

Esse estudo buscou realizar uma revisão integrativa da literatura (nacional e internacional) sobre o tema da resiliência comunitária, no período de 2009 a junho de 2015, com vistas a identificar o estado da arte sobre o tema, sobretudo no que toca a três aspectos: o conceito de resiliência comunitária, as principais adversidades estudadas e os fatores que favorecem resiliência comunitária. Espera-se que a realização dessa revisão integrativa possa lançar uma luz importante sobre o tema e motivar o desenvolvimento de estudos empíricos posteriores que considerem as adversidades e processos de resiliência comunitária específicos ao cenário nacional, tais como enchentes, seca, incêndios e desigualdade social.

\section{Método}

Trata-se de uma Revisão Integrativa da Literatura, a qual tem como objetivo reunir, avaliar criticamente e sintetizar, de maneira sistemática, os resultados de múltiplos estudos sobre um 
determinado tema, contribuindo para aprofundamento do conhecimento acerca da questão investigada (Mendes, Silveira, \& Galvão, 2008). Para nortear e sistematizar a execução da revisão integrativa da literatura percorreu-se oito etapas a seguir: (1) identificação do tema e delimitação da questão norteadora; (2) escolha das bases de dados; (3) definição dos descritores para a busca; (4) busca e armazenamento dos resultados; (5) seleção dos artigos pelo resumo, de acordo com os critérios de inclusão e exclusão; (6) obtenção dos dados dos artigos selecionados; (7) avaliação dos artigos; e 8) síntese e interpretação dos dados.

\section{Procedimentos}

Para realizar a busca, elegeu-se o Portal de Periódicos Capes, que oferece acesso a artigos indexados em diversas bases de dados, e a base SciELO. Em português foi utilizado o descritor "resiliência comunitária" e em inglês o descritor "community resilience". A busca nas bases estabeleceu o período entre 2009 e junho de 2015, com o intuito de mapear a produção mais recente (últimos sete anos), bem como por serem posteriores ao importante artigo publicado por Norris et al. (2008).

A pesquisa nas bases de dados ocorreu nos meses de maio e junho de 2015 e utilizou os seguintes critérios de inclusão: artigos indexados; publicados em periódicos revisados por pares; redigidos nos idiomas português, inglês, espanhol; artigos teóricos e empíricos; artigos que possuem o termo "resiliência comunitária" no título e/ou palavras chave; disponíveis em sua versão completa; publicados entre os anos de 2009 a junho de 2015; e realizada com seres humanos. Optou-se por privilegiar o uso do descritor "resiliência comunitária" e somente ele, bem como de incluir apenas artigos que apresentassem o referido termo no título ou palavras-chave, afim de melhor delimitar a busca e frisar aqueles estudos que investigam a resiliência comunitária, nomeando como tal o processo de superação de adversidades vividas no nível comunitário.

Posteriormente foram analisados os resumos (abstracts), considerando-se os seguintes critérios de exclusão: artigos duplicados; "literatura cinza" - capítulos de livro, notícias, documentos técnicos, comentários, editoriais, dissertações e teses; publicados fora do período entre 2009 e junho de 2015; estudos que não tivessem objetivos relacionados aos indicadores de resiliência comunitária. Após a seleção realizada pelo exame dos resumos, os artigos completos foram recuperados e submetidos à avaliação de três juízes independentes - todas estudantes de pós-graduação e integrantes do grupo de pesquisa do qual as autoras desse artigo também fazem parte. As exclusões foram esclarecidas por elas e as divergências resolvidas em consenso. Por fim, os estudos selecionados foram lidos na íntegra e a extração de dados foi armazenada em planilhas de Excel.

\section{Análise dos Dados}

A fim de obter a síntese e interpretação dos resultados foram realizadas dois tipos de análises. Na primeira, de natureza quantitativa (através do cálculo de frequências), buscou-se traçar o perfil da produção científica, sobretudo no que se refere aos seguintes indicadores: ano de publicação, idioma, nacionalidade das instituições dos primeiros autores, periódicos, natureza do artigo (empírico ou teórico), abordagem (quantitativa, qualitativa ou multimétodos) e instrumentos de coleta de dados. Na segunda análise, de natureza qualitativa, os conteúdos foram organizados e sintetizados, por meio da Análise de Conteúdo de Bardin (1977) e emergiram as seguintes categorias temáticas: definições de resiliência comunitária, os contextos de adversidade relatados nos artigos e os fatores que favorecem resiliência comunitária.

\section{Resultados e Discussão}

A busca inicial nas bases de dados foi realizada com filtros delimitando o período de publicação (2009-2015) e incluindo apenas publicações em periódicos revisados por pares. Identificou-se um total de 417 estudos. Diante da leitura dos resumos (abstracts), aplicaram-se os demais critérios de inclusão e exclusão, chegando-se aos seguintes dados: artigos duplicados 
$(n=122)$; pesquisas com não humanos $(n=45)$; publicações que não são artigo $(n=24)$; artigos que não possuem o termo "resiliência comunitária" ou "community resilience" no título e/ou palavras-chave $(n=135)$; estudos que não tinham o objetivo relacionado aos indicadores de resiliência comunitária ( $n=27)$. Após essa seleção, chegou-se ao número de 64 estudos, dos quais foi possível recuperar o texto completo de 61 , que foram lidos na íntegra pela pesquisadora e avaliados pelas juízas. Diante da avaliação, 24 artigos foram excluídos, pois não traziam em seu

417 Registros identificados através de pesquisa em bancos de dados (publicados no período entre 2009 e junho de 2015 em periódicos revisados por pares) conteúdo contribuições que ajudassem a responder às perguntas de partida da pesquisa, restando, portanto, 37 artigos que compuseram o corpo de análise desse estudo. O maior número de artigos foi obtido na base de dados PsycInfo $(n=$ 8), seguido pela John Wiley \& Sons, Inc $(n=7)$, Science Direct $(n=5)$, Pubmed e SciELO, ambas com 4 artigos, Sage Publications e Springer Science \& Business Media B.V. com 2 artigos, e, por fim, PsycArticles, ERIC, Proquest, National Library of Medicine e Scopus com 1 artigo cada. A Figura 1 sumariza o percurso metodológico da seleção dos artigos.

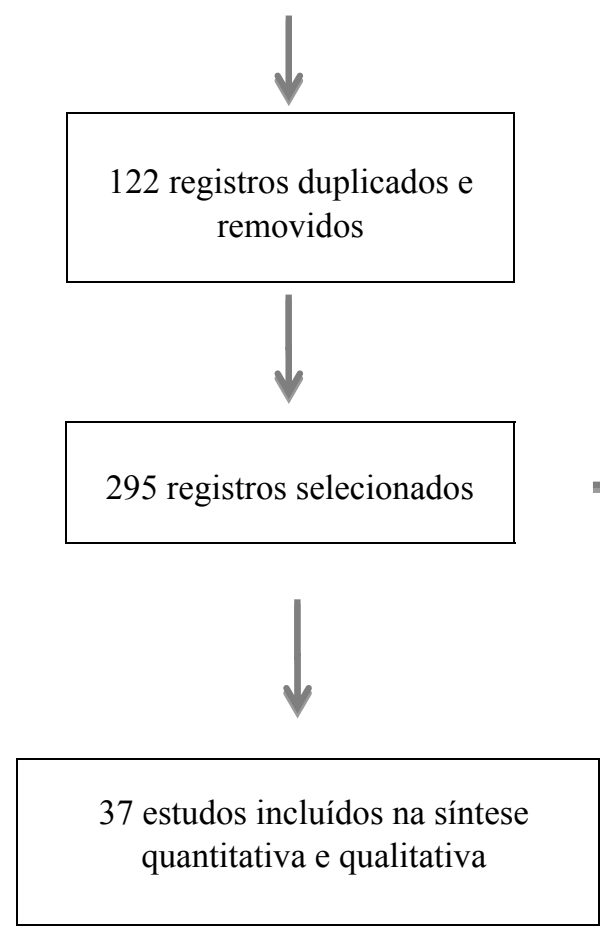

234 artigos excluídos após leitura dos resumos. Razões:

- não humanos $(\mathrm{n}=45)$;

- literatura cinza $(n=24)$;

- artigos que não possuem o termo "resiliência comunitária" no título e/ou nas palavras-chave $(\mathrm{n}=135)$;

- estudos que não tratam predominantemente de RC (n=27);

- artigos não disponíveis para download $(\mathrm{n}=3)$

24 artigos excluídos após leitura na íntegra e avaliação

Figura 1. Diagrama do processo de seleção dos artigos de acordo com os critérios de inclusão e exclusão

\section{Caracterização Quantitativa das Produções Científicas}

Entre os artigos analisados, a maioria dos estudos foi redigida no idioma inglês $(n=33)$, havendo três estudos em espanhol $(n=3)$ e um em português $(n=1)$. Quanto às instituições de origem dos autores dos artigos, a maioria é pro- veniente dos Estados Unidos $(n=17)$, em seguida Reino Unido $(n=7)$ e Austrália $(n=3)$. Dos 37 artigos encontrados, apenas 4 são de origem latino-americana, o que pode ser atribuído à falta de alcance dos artigos provenientes dessa região às revistas indexadas nas bases de dados selecionadas. Uma hipótese é a de que a escassez de 
estudos brasileiros e latino-americanos seja um indicador de que, nestes países, o aporte conceitual para o estudo dos processos organizativos e das potencialidades e desafios enfrentados pelas comunidades seja outro, como por exemplo, a Psicologia da Libertação, amplamente divulgada e trabalhada na Psicologia Comunitária latino-americana.

Observa-se que o número de publicações sobre resiliência comunitária vem crescendo com o passar dos anos, atingindo um número mais elevado em $2013(n=11)$. No que diz respeito às questões metodológicas, predominaram os artigos empíricos $(n=23)$. Dentre esses estudos, a abordagem qualitativa foi a mais utilizada $(n=13)$. Percebe-se grande diversidade nas estratégias de coletas de dados empíricos para acessar resiliência comunitária, sendo que muitos dos estudos fazem uso de mais de um instrumento. A entrevista foi o instrumento de coleta utilizado com maior frequência nos estudos selecionados $(n=13)$. O acesso a dados públicos para fins de pesquisa, como dados demográficos, arquivos históricos, conteúdo publicado online e dados de pesquisas realizadas anteriormente também foi bastante utilizado $(n=11)$.

Tabela 1

Caracterização Quantitativa dos Estudos Recuperados ( $n=37)$

\begin{tabular}{|c|c|}
\hline Categorias Quantitativas & Amostra \\
\hline \multirow{3}{*}{ Idioma } & Inglês $(n=33)$ \\
\hline & Espanhol $(n=3)$ \\
\hline & Português $(n=1)$ \\
\hline \multirow{6}{*}{ País de origem do primeiro autor } & Estados Unidos $(n=17)$ \\
\hline & Reino Unido $(n=7)$ \\
\hline & Austrália $(n=3)$ \\
\hline & Chile $(n=2)$ \\
\hline & Brasil, Canadá, Espanha, Israel, Líbano, México, Noruega e Sri \\
\hline & Lanka $(n=1)$ \\
\hline \multirow{7}{*}{ Ano de publicação } & $2009(n=1)$ \\
\hline & $2010(n=3)$ \\
\hline & $2011(n=4)$ \\
\hline & $2012(n=7)$ \\
\hline & $2013(n=11)$ \\
\hline & $2014(n=6)$ \\
\hline & Junho de $2015(n=5)$ \\
\hline \multirow{2}{*}{ Tipo de estudo } & Empírico $(n=23)$ \\
\hline & Teórico $(n=14)$ \\
\hline \multirow{3}{*}{ Método } & Qualitativo $(n=13)$ \\
\hline & Multimétodos $(n=5)$ \\
\hline & Quantitativo $(n=5)$ \\
\hline \multirow{5}{*}{ Instrumento } & Entrevista $(n=13)$ \\
\hline & Dados públicos $(n=11)$ \\
\hline & Questionários/escalas/índices $(n=7)$ \\
\hline & Observações em campo $(n=6)$ \\
\hline & Estratégias grupais $(n=4)$ \\
\hline
\end{tabular}




\section{Caracterização Qualitativa das Produções Científicas}

\section{O conceito de resiliência comunitária. A} análise dos artigos evidenciou que não existe um consenso com relação ao conceito de resiliência comunitária. Diversas são as definições encontradas, sendo que muitos artigos descrevem mais de uma definição ao fazer uma revisão introdutória, no caso dos estudos empíricos, ou uma revisão de literatura mais ampla, no caso dos estudos teóricos. O modelo teórico mais citado nos artigos foi o proposto por Norris et al. (2008), como resultado da vasta revisão de literatura publicada por esses autores, no qual a resiliência comunitária é vista como um processo que emerge de conjuntos de capacidades adaptativas interligadas. Dois (Azkarraga, Sloan, Belloy, \& Loyola, 2012; Juliano \& Yunes, 2014) dos artigos selecionados usaram como referência o quadro conceitual proposto por Ojeda (2005) e Ojeda et al. (2007), que descreve a resiliência comunitária como os processos e forças coletivas que permitem a metabolização do evento negativo e a mobilização de capacidades solidárias em direção à reconstrução.

Diante dos efeitos devastadores que algumas catástrofes geraram nos últimos anos, como o furacão Katrina em 2005 e os ataques terroristas de 11 de setembro de 2001, ambos nos Estados Unidos, a resiliência comunitária passou a ser do interesse de diversos países, integrando políticas nacionais de segurança e saúde pública em alguns deles, incluindo Estados Unidos (Plough et al., 2013) e Austrália (Boon et al., 2012). Nessa perspectiva, diversos estudos abordam a resiliência comunitária aplicada ao comportamento organizacional eficaz na gestão de desastres/ emergências (e.g. Harte, Childs, \& Hastings, 2009; White et al., 2014). Assim, tais estudos associam o conceito de resiliência comunitária às fases empregadas na gestão de emergências: resposta, recuperação e preparação. A resposta diz respeito à manutenção de serviços de emergência para lidar com os efeitos imediatos do evento catastrófico. Recuperação é o momento em que setor público e privado trabalham juntos na tentativa de alcançar um retor- no ao modo de vida anterior e oferecer segurança à área afetada. Já a preparação implica o desenvolvimento de ações que ajudam a minimizar os efeitos destrutivos desses eventos caso eles ocorram novamente, ou seja, ações que possibilitem futura resiliência comunitária (Rob, 2012).

Em alguns estudos, o conceito de resiliência comunitária é associado ao de resiliência inerente, que consiste em práticas arquivadas na memória coletiva utilizadas pela comunidade para lidar com rupturas (Colten, Hay, \& Giancarlo, 2012). Os estudos nessa perspectiva descrevem resiliência comunitária como a habilidade de um sistema humano de absorver o impacto e se reorganizar, mantendo a mesmo função, estrutura e identidade enquanto passa por graves mudanças (González-Muzzio, 2013; Wilson, 2013b). Para tanto, a comunidade precisa contar tanto com condições inerentes ao sistema que lhe permitam enfrentar o evento, como com os processos adaptativos posteriores que facilitam a capacidade do sistema de se reorganizar, mudar e aprender em resposta ao evento (González-Muzzio, 2013). Portanto, a comunidade é competente para enfrentar as adversidades, mas precisa do apoio e encorajamento adequados para conseguir acessar as forças coletivas que permitirão transcender a perda (Agani, Landau, \& Agani, 2010).

A resiliência comunitária tem sido compreendida também em termos de recursos ou capitais (social, físico, econômico e humano) que a comunidade possui e que podem ser acessados em um momento de crise. De acordo com Ungar (2011), resiliência comunitária consiste na relação de interdependência entre o capital social, a infraestrutura física e os padrões culturalmente incorporados que dão à comunidade o potencial para recuperar-se de uma mudança dramática, sustentar sua capacidade de adaptação e apoiar um novo crescimento que integra as lições aprendidas com a crise. Assim, recuperação, sustentabilidade e crescimento são possíveis, mas é preciso que existam forças comunitárias nutridas antes do evento estressor, recursos significativos disponíveis para a comunidade oferecidos pelo governo e capacidade comunitária para usá-los em benefício comum (Ungar, 2011). 
De acordo com a análise das publicações, a resiliência comunitária é vista, portanto, como atributo (capacidade, habilidade) e enquanto processo. As definições que usam o termo capacidade, em geral, são aquelas relacionadas à gestão de emergências e à possibilidade de uma comunidade oferecer os recursos necessários ao enfrentamento da adversidade somada à capacidade dos indivíduos de acessá-los. Já o termo habilidade é mais utilizado em uma perspectiva ecológica, na qual a ação coletiva permite absorver a perturbação, se reorganizar e manter essencialmente a mesma função, enquanto passa por uma mudança. Por fim, a definição de resiliência comunitária enquanto processo está relacionada à adaptação positiva da comunidade frente a uma situação de desastre ou crise. Nesse caso, o desfecho ou resultado seria a adaptação (indicada pelo bem-estar comunitário), enquanto a resiliência seria o processo que levou a comunidade a esse desfecho, incluindo a relação de interdependência entre os recursos sociais, culturais e econômicos, que fornece o potencial para a comunidade lidar da forma mais positiva possível com a adversidade.

Contextos adversos. Diversos contextos adversos vivenciados por comunidades são descritos nos artigos selecionados. Grande parte dos estudos teóricos abordam os desastres de uma forma geral, tanto os de causa natural (furacões, terremotos, enchentes) como os causados pelo homem (ataques terroristas, conflitos armados) enquanto estressores comunitários (e.g. Ungar, 2011). Os desastres são eventos extremos, geralmente repentinos e inesperados, experienciados individual e coletivamente. Eles causam ferimento, morte, destruição de recursos físicos e econômicos, provocam caos, interrompem rotinas e ameaçam gravemente o funcionamento das comunidades (Pfefferbaum \& Pfefferbaum, 2013). Alguns desses estudos problematizam o aumento da população vulnerável a esses desastres, sublinhando a necessidade de se pensar em formas de reduzir essa vulnerabilidade antes da ocorrência do desastre (González-Muzzio, 2013; Harte et al., 2009).

Diversos estudos dão ênfase aos desastres naturais no contexto da mudança climática, dis- cutindo as tendências atuais globais, tais como aumento da temperatura atmosférica, elevação do nível do mar, que tem como consequência uma maior probabilidade de ocorrência dos desastres naturais. Estão interessados, portanto, na complexa relação entres os impactos da mudança climática e as respostas sociais que as comunidades apresentam, mais especificamente a capacidade de adaptar-se com sucesso a essas mudanças (e.g. Gómez-Baggethun, Reyes-García, Olsson, \& Montes, 2012; Smith et al., 2012).

Além disso, discute-se a ameaça de perda da resiliência das comunidades devido à rápida globalização mundial que tem como consequência vulnerabilidade econômica, social e ambiental. A inserção da maioria das comunidades no sistema capitalista global dificulta os processos de resiliência para algumas delas, pois nem sempre é possível encontrar um balanço entre a comunidade e suas interações a nível global. É arriscado para uma comunidade tanto um isolamento que pode levá-la a uma completa dependência de seus recursos locais, quanto uma exagerada globalização que pode resultar em perda de autonomia e identidade (Wilson, 2012, 2013a). Nessa perspectiva, dois artigos abordam resiliência comunitária no contexto de comunidades indígenas, uma situada no Alaska, Estados Unidos e a outra no Nordeste da Noruega, que enfrentam dificuldades por seus jovens crescerem em um contexto muito diferente de seus pais e avós, devido às rápidas mudanças sociais, econômicas, culturais, políticas e ecológicas, que resultam em graves problemas sociais (Nystad, Spein, \& Ingstad, 2014; Wexler, Joule, Garoutte, Mazziotti, \& Hopper, 2013).

Os contextos de pobreza e privação de recursos são discutidos em dois artigos, ambos publicados em revistas da área da saúde. Eles discutem a relação entre bairros desfavorecidos (deprived neighborhood), saúde e resiliência comunitária (Allmark, Bhanbhro, \& Chrisp, 2014; Poortinga, 2012). De acordo com esses estudos, a ideia de que resiliência comunitária pode contribuir para saúde pública com relação a ameaças de longo prazo, como a pobreza, faz parte do desenvolvimento de estudos mais recentes sobre o tema. Poortinga (2012) afirma que um bairro 
com grande privação de recursos representa um dos mais importantes fatores de risco para saúde pública e bem-estar, pois limita o acesso à educação e às oportunidades de emprego, aumenta a experiência de estresse e pode afetar a autoestima e o suporte social.

Desse modo, percebe-se que predominam os estudos discutindo as adversidades relacionadas aos desastres e emergências. Menor ênfase é dada às outras questões, como os desafios lançados pela globalização e a vulnerabilidade causada pela pobreza e privação de recursos. Apesar de poucos artigos focarem tais aspectos, podem apontar para o início dos estudos de resiliência comunitária em outros campos de aplicação do conceito que ainda não foram muito explorados.

Fatores que favorecem resiliência comunitária. Aspectos sociais encontrados na comunidade apareceram com maior frequência nos resultados dos estudos como aqueles fatores que indicam resiliência comunitária ou contribuem para seu desenvolvimento. O capital social tem sido amplamente discutido na literatura e há um consenso sobre o importante papel que exerce nos processos de resiliência comunitária. Instituições governamentais dos Estados Unidos e Reino Unido têm realizado alto investimento financeiro em pesquisas e políticas públicas focando a infraestrutura física no processo de gestão de emergências (Aldrich \& Meyer, 2015). No entanto, os autores que discutem o capital social concordam que os esforços empreendidos na preparação para desastres devem ser mais focados na infraestrutura social, pois é esta que impulsiona resiliência comunitária (Aldrich \& Meyer, 2015; Poortinga, 2012; Rob, 2012).

O Capital Social é formado pelas redes de suporte social que o indivíduo pode acessar, sejam redes informais (amigos, vizinhos e familiares) ou estruturas formais de apoio social, como equipamentos sociais e ONGs (Aldrich \& Meyer, 2015; Poortinga, 2012; Ungar, 2011). Este construto é refletido através de confiança social, reciprocidade e participação civil e vem sendo relacionado a altos níveis de saúde subjetiva, melhor saúde mental e redução dos efeitos adversos de viver em bairros desfavorecidos (Poortinga, 2012).
O Capital Social tem sido separado em três tipos principais: bonding, bridging e linking. Cada um varia de acordo com a força dos relacionamentos, a composição da rede e os diferentes desfechos para indivíduos e comunidades. Bonding diz respeito às conexões existentes entre indivíduos próximos emocionalmente, como amigos, vizinhos e familiares. A forte ligação fornece aos indivíduos coesão social, suporte e assistência pessoal quando eles necessitam, especialmente em situações de crise. Bridging descreve as relações sociais mais distantes, entre conhecidos ou indivíduos frouxamente conectados, mas que pertencem a um mesmo grupo, incluindo raça, classe social ou organizações (grupos religiosos, associação de pais e professores e instituições civis e políticas). Tal ligação pode contribuir para o avanço de determinado grupo na sociedade, através da solidariedade, respeito e compreensão entre indivíduos heterogêneos. $\mathrm{O}$ terceiro tipo de capital social, linking, conecta os cidadãos aos seus governantes. Este tipo de rede incorpora normas de respeito e relações de confiança com autoridades, sejam elas formais ou institucionalizadas em sociedade, que permitem a mobilização de recursos políticos (Aldrich \& Meyer, 2015; Poortinga, 2012).

Alguns aspectos do capital social são discutidos nos artigos, mesmo que não se refiram a este termo. $\mathrm{O}$ apoio encontrado nas relações familiares, que correspondem a uma parte do capital social bonding, é considerado de grande importância para a resiliência comunitária por alguns autores (e.g. Agani et al., 2010; Colten et al., 2012; Nystad et al., 2014). Segundo Agani et al. (2010), conectividade com a família e cultura de origem está relacionada com redução de comportamento de risco, violência social e estresse pós-traumático. Promover o fortalecimento dos laços familiares e melhorar o acesso dos indivíduos à família estendida, portanto, pode ser protetivo em situações de estresse. Além disso, a interconectividade com os demais membros da comunidade e deles para com o meio ambiente foram apontadas como promotoras de resiliência e bem-estar (Nystad et al., 2014). Uma vez que o capital social pode ser gerado ou degradado (desgastado), os esforços nesse sentido devem 
se voltar para reforçar a coesão social e aprofundar a confiança nas comunidades, construindo pontes entre grupos dentro da comunidade e destes com aqueles que têm autoridade (Aldrich \& Meyer, 2015).

Os laços culturais envolvendo determinado grupo tem se mostrado de grande importância para a resiliência comunitária. $\mathrm{O}$ estudo sobre a guerra do Líbano de 2006 concluiu que o sentido de uma identidade coletiva e experiência prévia com guerras favoreceram a construção de resiliência comunitária ao longo do tempo (Nuwayhid, Zurayk, Yamout, \& Cortas, 2011). Assim, costumes locais, resiliência inerente, orgulho étnico, práticas e rituais tradicionais, crenças e normas parecem contribuir para os processos de resiliência comunitária (Gómez-Baggethun et al., 2012; Nuwayhid et al., 2011; Nystad et al., 2014; Wilson, 2013b).

Apesar de não receber grande atenção nos estudos selecionados nessa revisão, a infraestrutura física de uma comunidade, no que diz respeito ao acesso a transporte público, espaços de lazer, saúde, educação e suporte social de qualidade também são considerados fatores promotores de resiliência. De acordo com Ungar (2011), quando as pessoas que mais necessitam desse tipo de suporte têm acesso a ele, alguns riscos são minimizados. Assim, um transporte público de qualidade pode facilitar que jovens da periferia tenham acesso a oportunidades de emprego e aos serviços de saúde; espaços de lazer podem facilitar a aproximação entre membros da comunidade; e o acesso à educação amplia as possibilidades de desenvolvimento dos indivíduos e, consequentemente, da comunidade.

É possível perceber semelhanças entres os fatores encontrados nos artigos analisados nessa revisão, em sua maioria considerando o modelo de Norris et al. (2008) de resiliência comunitária, e os pilares da resiliência comunitária propostos por Ojeda (2005). O capital social abarca os pilares solidariedade (ações coletivas que buscam o bem comum da comunidade) e honestidade estatal (relação de confiança entre moradores e governantes). Já a identidade cultural, humor social e autoestima coletiva dizem respeito à relação dos membros com o lugar de origem, compondo os laços sociais. A tentativa de integração dos resultados dessa revisão com a contribuição latino-americana para o conceito de resiliência é sumarizada na Tabela 2.

Tabela 2

Fatores que Favorecem Resiliência Comunitária a partir da Integração do Modelo Norte-Americano e Latino-Americano

Fatores Definição Exemplos de Referências

Suporte social informal - laços de solidariedade entre familiares, vizinhos e amigos;

Suporte social formal - equipamentos sociais e ONGs;

Grupos sociais - raça, classe social, associações;

(Agani et al., 2010; Allmark et al., 2014; González-Muzzio, 2013; Organização comunitária - liderança, coesão social, participação comunitária;

Juliano \& Yunes, 2014; Poortinga, 2012; Wyche et al., 2011)

Capital Social Relação de confiança entre moradores e seus governantes

Identidade Cultural - costumes e crenças compartilhados; Conhecimento acumulado de experiências anteriores; Autoestima coletiva - amor e orgulho pelo lugar

(Colten et al., 2012;

Gómez-Baggethun et al., 2012; González \& Gaudino, 2013; Juliano

Laços Culturais de origem; Humor Social \& Yunes, 2014; Nystad et al., 2014;

G. A. Wilson, 2013b)

Saúde; Educação; Segurança; Transporte público; Espaços de lazer

(González \& Gaudino, 2013; Ungar, 2011) 


\section{Considerações Finais}

Este artigo teve como objetivo realizar uma revisão integrativa da literatura (nacional e internacional) sobre o tema da resiliência comunitária, no período de 2009 a junho de 2015, com vistas a identificar o estado da arte acerca do tema, especificamente no que diz respeito ao conceito, principais adversidades estudadas e fatores que favorecem resiliência comunitária.

De acordo com o observado na análise quantitativa, as publicações sobre Resiliência Comunitária cresceram significativamente somente no ano de 2013, o que evidencia quão recente é a discussão sobre esse tema. Apesar de novo, o tema apresenta grande potencial de contribuição para se pensar a superação de adversidades no nível comunitário. Embora seja possível o acesso ao modelo latino-americano de resiliência comunitária através de livros, apenas quatro artigos originados desse continente foram encontrados nesta busca, o que confirma a necessidade de publicações de origem latina acerca desse tema em revistas de maior impacto e visibilidade. Ademais, entende-se que o baixo número de publicações identificadas, sobretudo de estudos latino-americanos, pode estar relacionada ao uso exclusivo do descritor "resiliência comunitária" no título ou palavras-chave, à limitação de bases de dados latino-americanas incluídas na busca, bem como a restrição da busca a artigos, ao invés de livros e capítulos de livros. Certamente, a inclusão de outros descritores (e.g. adaptação, bem-estar comunitário, etc.) levaria à inclusão de um maior número de estudos. Da mesma forma, a inclusão de outras bases na busca, tais como IndexPsi, Pepsic e BVS-Psi, somada à consideração de livros e capítulos de livros, poderia ampliar os resultados.

Tal consideração, no entanto, não exclui a hipótese de que, nos países latino-americanos, conforme já mencionado na introdução desse artigo, o aporte conceitual utilizado para estudar as adversidades e processos de superação comunitários seja outro. Nesse caso, estudos futuros poderiam focar-se na discussão de como a área da Psicologia Comunitária tem entendido e trabalhado - de forma direta ou indireta - o conceito de resiliência comunitária. No entanto, reitera-se aqui a necessidade de uma clareza acerca do conceito de resiliência comunitária, de forma a não se nomear como resiliência, conceitos/processos que podem ser nomeados de outra maneira.

Verificou-se que as principais adversidades estudadas são os desastres de causa natural ou humana. Em menor número, encontram-se estudos focando os desafios lançados pelas mudanças ambientais e globalização, que resultam, por exemplo, em dificuldades de crescer em uma comunidade indígena e em riscos gerados pela pobreza e privação de recursos. Portanto, um campo vasto de pesquisa e intervenção, voltado às situações de adversidades crônicas, como as vividas no contexto latino-americano (e.g. enchentes, secas, violência, pobreza etc) está aberto aos pesquisadores e profissionais interessados em resiliência comunitária.

No que toca aos recursos necessários para que ocorram processos de resiliência comunitária, destacam-se o capital social, infraestrutura e laços culturais. Tais fatores resultam de uma análise integradora, proposta nesse artigo, a qual buscou avançar ao estabelecer pontos de convergência entre os pilares da resiliência comunitária, tradicionalmente enfatizados na literatura latino-americana e norte-americana. Assim sendo, sugere-se que estudos futuros podem caminhar no sentido de uma perspectiva integradora da resiliência comunitária. Além disso, acredita-se ser importante o maior desenvolvimento de estudos empíricos sobre resiliência comunitária no contexto brasileiro, somado ao desenvolvimento e avaliação de intervenções psicossociais, promotoras de resiliência comunitária.

\section{Referências}

Agani, F., Landau, J., \& Agani, N. (2010). Community-building before, during, and after times of trauma: The application of the linc model of community resilience in kosovo. American Journal of Orthopsychiatry, 80(1), 143-149. doi: http:// doi.org/10.1111/j.1939-0025.2010.01017.x

Aldrich, D., \& Meyer, M. (2015). Social Capital and Community Resilience. American Behavioral Scientist, 59(2), 254-269. doi: http://doi. org/10.1177/0002764214550299 
Allmark, P., Bhanbhro, S., \& Chrisp, T. (2014). An argument against the focus on community resilience in public health. BMC Public Health, 14, 62. doi: http://doi.org/10.1186/1471-2458-14-62

Azkarraga, J., Sloan, T., Belloy, P., \& Loyola, A. (2012). Eco-localismo y resiliencia comunitaria frente a la crisis civilizatoria. Polis, 33, 19. Recuperado en http://journals.openedition.org/ polis/8400

Bardin, L. (1977). Análise de conteúdo (L. A. Reto \& A. Pinheiro, Trads.). Lisboa: Edições 70.

Boon, H. J., Cottrell, A., King, D., Stevenson, R. B., \& Millar, J. (2012). Bronfenbrenner's bioecological theory for modelling community resilience to natural disasters. Natural Hazards, 60(2), 381-408. doi: http://doi.org/10.1007/ s11069-011-0021-4

Coimbra, R., \& Morais, N. (2015). A resiliência em questão: Perspectivas teóricas, pesquisa e intervenção. Porto Alegre, RS: Artmed.

Colten, C. E., Hay, J., \& Giancarlo, A. (2012). Community resilience and oil spills in coastal Louisiana. Ecology and Society, 17(3). doi: http://doi. org/10.5751/ES-05047-170305

Góis, C. W. de L. (2008). Saúde comunitária: Pensar e fazer. São Paulo, SP: Aderaldo \& Rothschild.

Gómez-Baggethun, E., Reyes-García, V., Olsson, P., \& Montes, C. (2012). Traditional ecological knowledge and community resilience to environmental extremes: A case study in Doñana, SW Spain. Global Environmental Change, 22(3), 640-650. doi: http://doi.org/10.1016/j. gloenvcha.2012.02.005

González, A. L. M., \& Gaudino, E. F. G. (2013, August). De la resiliencia comunitaria a la ciudadanía ambiental. Revista Integra Educativa, 6(3), 13-28. Recuperado en http://www.scielo.org.bo/scielo.php?script=sci_arttext\&pid $=$ S1997-40432013000300002

González-Muzzio, C. (2013). El rol del lugar y el capital social en la resiliencia comunitaria posdesastre. Aproximaciones mediante un estudio de caso después del terremoto del 27/F. Eure, 39(117), 25-48. doi: http://doi.org/10.4067/ S0250-71612013000200002

Harte, E. W., Childs, I. R. W., \& Hastings, P. A. (2009). Imizamo Yethu: A case study of community resilience to fire hazard in an informal settlement Cape Town, South Africa. Geographical Research, 47(2), 142-154. doi: http://doi. org/10.1111/j.1745-5871.2008.00561.x
Juliano, M. C., \& Yunes, M. A. (2014). Reflexões sobre rede de apoio social como mecanismo de proteção e promoção de resiliência. Ambiente \& Sociedade, 17(3), 135-154.

Kirmayer, L. J., Sehdev, M., Whitley, R., Dandeneau, S. F., \& Isaac, C. (2009). Community Resilience: Models, Metaphors and Measures. International Journal of Indigenous Health, 5(1), 62-117. doi: http://doi.org/10.1073/pnas.0803780105

Mendes, K. D. S., Silveira, R. C. D. C. P., \& Galvão, C. M. (2008). Revisão integrativa: Método de pesquisa para a incorporação de evidências na saúde e na enfermagem. Texto \& Contexto - Enfermagem, 17(4), 758-764. doi: http://doi. org/10.1590/S0104-07072008000400018

Montero, M. (1984). La psicología comunitaria: orígenes, principios y fundamentos teoricos. Revista Latinoamericana de Psicología, 16(3), 387400.

Morais, N. A., \& Koller, S. H. (2011). Abordagem Ecológica do Desenvolvimento Humano, Psicologia Positiva e Ciência: Ênfase na Saúde. In Ecologia do desenvolvimento humano: Pesquisa e intervenção no Brasil. São Paulo, SP: Casa do Psicólogo.

Morais, N., Figueiredo, D., Rodrigues, T., \& Santos, R. (2015). "Mandacaru quando fulora na seca": Um estudo sobre processos de resiliência vividos por moradores do sertão cearense. In $\mathrm{R}$. Coimbra \& N. Morais (Eds.), A resiliência em questão: Perspectivas teóricas, pesquisa e intervenção. Porto Alegre, RS: Artmed.

Norris, F. H., Stevens, S. P., Pfefferbaum, B., Wyche, K. F., \& Pfefferbaum, R. L. (2008). Community resilience as a metaphor, theory, set of capacities, and strategy for disaster readiness. American Journal of Community Psychology, 41(1-2), 127-150. doi: http://doi.org/10.1007/s10464007-9156-6

Nuwayhid, I., Zurayk, H., Yamout, R., \& Cortas, C. S. (2011, March). Summer 2006 war on Lebanon: A lesson in community resilience. Global Public Health, 6, 505-519. doi: http://doi.org/10 $.1080 / 17441692.2011 .557666$

Nystad, K., Spein, A. R., \& Ingstad, B. (2014). Community resilience factors among indigenous Sámi adolescents: A qualitative study in Northern Norway. Transcultural Psychiatry, 51(5), 651-672. doi: http://doi.org/10.1177/1363461514532511

Ojeda, E. (2005). Uma concepção latino-americana: A resiliência comunitária. In A. Melillo \& E. 
Ojeda (Eds.), Resiliência: Descobrindo as próprias fortalezas (pp. 47-57). Porto Alegre, RS: Artmed.

Ojeda, E., La Jara, A., \& Márquez, C. (2007). Resiliência Comunitária. In L. Hoch \& S. Rocca (Eds.), Sofrimento, resiliência e fé: Implicações para as relações de cuidado (pp. 33-56). São Leopoldo, RS: Sinodal.

Pfefferbaum, R. L., \& Pfefferbaum, B. (2013). Community resilience to disasters for mental health professionals. Direcitions in Psychiatry, 33(2), 135-145.

Plough, A., Fielding, J. E., Chandra, A., Williams, M., Eisenman, D., Wells, K. B., ...Magaña, A. (2013). Building Community Disaster Resilience: Perspectives from a Large Urban County Department of Public Health. American Journal of Public Health, 103(7), 1190-1197. doi: http:// doi.org/10.2105/AJPH.2013.301268

Poletto, M., \& Koller, S. H. (2008). Contextos ecológicos: Promotores de resiliência, fatores de risco e de proteção. Estudos de Psicologia (Campinas), 25(3), 405-416. doi: http://doi.org/10.1590/ S0103-166X2008000300009

Poortinga, W. (2012). Community resilience and health: The role of bonding, bridging, and linking aspects of social capital. Health and Place, 18(2), 286-295. doi: http://doi.org/10.1016/j. healthplace.2011.09.017

Quintal, M. de F. (2016). Desafios atuais e antigas sutilezas nas práticas da psicologia social comunitária. Psicología, Conocimiento Y Sociedad, 6(1), 131-163.

Rob, A. (2012). Building Community Resilience. Proceedings of the Institution of Civil Engineers, 165(CE6), 59-64.

Rutter, M. (1993). Resilience: Some Conceptual Considerations. Journal of Adolecent Health, 14(8), 626-631.

Smith, J. W., Moore, R. L., Anderson, D. H., \& Siderelis, C. (2012). Community Resilience in Southern Appalachia: A Theoretical Framework and Three Case Studies. Human Ecology, 40(3),
341-353. doi: http://doi.org/10.1007/s10745012-9470-y

Ungar, M. (2011). Community resilience for youth and families: Facilitative physical and social capital in contexts of adversity. Children and Youth Services Review, 33(9), 1742-1748. doi: http://doi.org/10.1016/j.childyouth.2011.04.027

Wexler, L., Joule, L., Garoutte, J., Mazziotti, J., \& Hopper, K. (2013). "Being responsible, respectful, trying to keep the tradition alive:" Cultural resilience and growing up in an Alaska Native community. Transcultural Psychiatry, 51(5), 693-712. doi: http://doi. org/10.1177/1363461513495085

White, R. K., Edwards, W. C., Farrar, A., \& Plodinec, M. J. (2014). A Practical Approach to Building Resilience in America's Communities. American Behavioral Scientist, 59(2), 200-219. doi: http://doi.org/10.1177/0002764214550296

Wilson, G. A. (2012). Community resilience, globalization, and transitional pathways of decision-making. Geoforum, 43(6), 1218-1231. doi: http://doi.org/10.1016/j.geoforum.2012.03.008

Wilson, G. A. (2013a). Community resilience, policy corridors and the policy challenge. Land Use Policy, 31, 298-310. doi: http://doi. org/10.1016/j.landusepol.2012.07.011

Wilson, G. A. (2013b). Community resilience, social memory and the post-2010 Christchurch (New Zealand) earthquakes. Area, 45(2), 207-215. doi: http://doi.org/10.1111/area.12012

Zappe, J., Yunes, M. A., \& Dell'aglio, D. (2015). Psicologia dos desastres e resiliência comunitária: Reflexões sobre o incêndio da boate Kiss em Santa Maria/RS. In R. M. Coimbra \& N. A. Morais (Eds.), A resiliência em questão: Perspectivas teóricas, pesquisa e intervenção. Porto Alegre, RS: Artmed.

Recebido: 23/05/2016

$1^{a}$ revisão: 07/05/2017

Aceite final: 02/09/2017

(c) BY (C) O(s) autor(es), 2018. Acesso aberto. Este artigo está distribuído nos termos da Licença Internacional Creative Commons Atribuição 4.0 (http://creativecommons.org/licenses/by/4.0/), que permite o uso, distribuição e reprodução sem restrições em qualquer meio, desde que você dê crédito apropriado ao(s) autor(es) original(ais) e à fonte, fornecer um link para a licença Creative Commons e indicar se as alterações foram feitas. 